\title{
Mondialisation et aide humanitaire dans
}

l'Himalaya népalais

Le global-théorique à l'épreuve du local-empirique

Lionel Obadia

\section{OpenEdition}

\section{Journals}

Édition électronique

URL : https://journals.openedition.org/pa/1875

DOI : $10.4000 /$ pa. 1875

ISSN : 2273-0362

\section{Éditeur}

Université Lumière Lyon 2

\section{Édition imprimée}

Date de publication : 1 janvier 2005

Pagination : 43-52

ISBN : 1634-7706

ISSN : 1634-7706

\section{Référence électronique}

Lionel Obadia, « Mondialisation et aide humanitaire dans l'Himalaya népalais ». Parcours

anthropologiques [En ligne], 5 | 2005, mis en ligne le 06 juillet 2021, consulté le 08 décembre 2022

URL : http://journals.openedition.org/pa/1875; DOI : https://doi.org/10.4000/pa.1875 


\section{MONDIALISATION ET AIDE HUMANITAIRE DANS L'HIMALAYA NÉPALAIS}

\section{LE GLOBAL-THÉORIQUE À L'ÉPREUVE DU LOCAL-EMPIRIQUE}

\section{Le Népal et la mondialisation ? Questions préalables}

Après s'être imposé dans les sciences politiques, en sociologie, et en histoire, le terme de mondialisation a récemment poursuivi sa longue marche au sein de l'anthropologie. L'accueil qui lui a été réservé n'est jusqu'ici pas toujours des plus enthousiastes. Dans un article récent, Mauro Guillèn a par exemple montré - chiffres à l'appui - que les publications mentionnant le vocable de mondialisation étaient, au cours de la précédente décennie, deux à trois cent fois plus nombreuses en sociologie et en politologie qu'en anthropologie ${ }^{1}$. Ce n'est que très récemment qu'on l'a vu gagner en popularité, sous l'impulsion notable des travaux d'Appadurai, de Hannerz ou de Friedman. Jusqu'à quel point ces concepts de portée aussi générale et de confection historicopolitologique tels que celui de mondialisation servent-ils (à) l'anthropologie ? Il y a déjà longtemps que l'on reconnaît à la discipline une ouverture aux idées et concepts issus d'autres matrices intellectuelles. Et que l'on s'accorde sur le fait que les concepts sont par définition nomades et ne sauraient se limiter à des cadres théoriques et disciplinaires exclusifs. Pour autant que l'anthropologie se montre aussi intégrative qu'on veuille bien le dire, les concepts empruntés à d'autres disciplines ne se transposent toutefois jamais en l'état d'un espace scientifique à l'autre. Ce déplacement de cadre convoque et réinterroge - ou du moins le devrait - les théories, l'épistémè et surtout la méthode de chaque discipline qui s'inspire d'un concept tel que celui de mondialisation. Qui est en outre le pivot d'une théorie générale du monde - une ambition que bien des approches actuelles en anthropologie ont abdiquée.

Ce qui fait en premier lieu problème, c'est l'emploi même du terme mondialisation: s'agit-il d'un vocable à usage purement nominal - dont le sens est alors essentiellement conventionnel - ou au contraire d'un concept stricto sensu qui implique alors un effort de définition? Le problème est d'autant plus épineux que personne ne semble s'accorder sur une définition minimale ${ }^{2}$. Faut-il ensuite le considérer au singulier - comme un phénomène unique dans ses sources, formes et manifestations - ou au pluriel - sous des expressions diverses qui ne se conjuguent au singulier qu'en vertu de leur simultanéité ? Autrement dit, est-il plus légitime de parler de mondialisation ou de mondialisations? Les options théoriques et méthodologiques susceptibles d'être retenues pour répondre à ces questions sont légion, et ce n'est pas ici le lieu de les énoncer toutes. Celles qui, en revanche, feront la preuve de leur opérationnalité scientifique ne nous paraissent pas légitimes si elles se fondent $a$ priori, presque plus, pourrait-on dire, sur le régime de la conviction ou de l'opinion que sur celui de la démonstration. C'est la raison pour laquelle je m'en tiendrai ici à une perspective résolument ancrée dans l'empirique (ou une grounded theory pour concéder un tant soit peu à la mode actuelle), en souscrivant à l'idée que le terrain est seul à même, sinon de répondre à certaines de ces questions, du moins de les aborder sans trop encourir le péril d'une focale exclusivement intellectualiste. Il s'agira alors d'interroger de manière malheureusement trop peu détaillée, format oblige - une certaine forme de mondialisation, vue unanimement dans la littérature spécialisée comme un de ses vecteurs essentiels, qu'est l'aide humanitaire.

Le Népal a connu dans un temps relativement court (un demi-siècle) une série de changements. Ceux-ci se lisent sur différents plans, en particulier celui de l'économique et $\mathrm{du}$ politique, deux dimensions par ailleurs largement mobilisées dans les Globalization Studies. L'histoire officielle veut qu'au début des années 1950, après la chute du régime rana qui s'était illustré par une politique de clôture des frontières et une volonté de limiter les influences extérieures (asiatiques et occidentales), le Népal aurait été brusquement exposé à l'économie de marché, au tourisme et à l'aide internationale. Et donc confronté à des processus qui sont généralement considérés comme constitutifs de la mondialisation. Mais le concept de mondialisation est-il le plus approprié pour rendre compte des transformation du Népal ? Jusqu'à une période récente, la notion de modernité était préférée à toute autre. Le Népal serait ainsi "entré dans la modernité » ou y aurait été "projeté » par sa brusque confrontation à un monde dont il aurait longtemps été détourné par la réduction de ses échanges avec d'autres pays. Cette vision, dont la réalité historique est discutable, met en fait en question une autre idée : celle de l'ontologie de la mondialisation. La permutabilité des termes «mondialisation" et "modernité " laisse en effet perplexe : s'agit-il de la même chose? De deux processus en interaction, comme le voudrait Giddens (1994) ? Ou encore de deux phénomènes historiques distincts? On abandonnera ici la critique de la modernité déjà débutée ailleurs ${ }^{3}$ pour se concentrer sur le statut de la mondialisation. Comment 
s'inscrit-elle et jusqu'à quel point transformet-elle un pays qui est déjà constitué, depuis des temps anciens de son histoire (comme région politiquement parcellisée) jusqu'à sa fondation récente comme royaume unifié, autour de «flux " culturels, structuré autour de réseaux locaux et transnationaux, de formes culturelles et d'expressions religieuses diversifiées, en bref, qui présente antérieurement à la dite mondialisation les caractéristiques qu'elle est supposée y occasionner?

\section{Les ONG au Népal : acteurs du changement, vecteurs de la mondialisation}

Agents essentiels du changement social et technologique, du développement sanitaire ou même de la continuité culturelle, pourtant rarement étudiés en tant que tels dans les travaux d'une anthropologie longtemps centrée sur les problématiques "classiques" de la discipline, les ONG ne sont apparues qu'assez tardivement et de manière très secondaire dans la littérature ethnologique sur le Népal ${ }^{4}$. Les agents du développement, qu'ils prennent la forme d'acteurs institutionnalisés (ou pas) de l'aide internationale ou d'exécutants des programmes de développement nationaux, ont pourtant fait irruption depuis maintenant près de quatre décennies dans les configurations sociales et culturelles étudiées par les ethnologues 5 . Les études sur le développement - commanditées par l'État ou issues de groupes de recherches universitaires ou indépendants, népalais ou étrangers - ont parallèlement connu des progrès rapides et massifs. Les positions critiques adoptées dans la plupart de ces études ont entre autres points dénoncé l'indifférence qu'ont respectivement témoignée les agences de développement et les ethnologues: les premières élaborant des programmes ou des actions de développement en négligeant délibérément les données sociales et culturelles produites par l'ethnologie (notamment les structurations en castes et les systèmes de représentations culturelles locaux $)^{6}$, les seconds déclinant trop souvent les invitations répétées qui leur ont été adressées à se transformer en "experts" pour les questions culturelles, dans les plans de développement ${ }^{7}$. Autrement dit, alors que l'aide humanitaire ou la coopération au développement est engagée dans sa propre mondialisation (en s'immisçant dans le réseau dense des relations internationales et en structurant certains de ses segments), l'anthropologie fait l'objet d'une mondialisation parallèle (parce qu'enseignée ou pratiquée dans une multitude de pays bien au-delà de l'Europe occidentale qui l'a vue naître) sans que l'une et l'autre ne se semblent disposées à se rencontrer.

Il est cependant loin d'être inhabituel que les acteurs de l'aide humanitaire se transforment en ethnologues au contact du terrain ${ }^{8}$. Les invitations adressées à l'anthropologie pour qu'elle s'intéresse à des questions qui s'inscrivent généralement dans les problématiques du développement (pauvreté, malnutrition, qualité de vie, rapport à l'environnement, rapports entre modes de production et systèmes sociaux, gestion des ressources naturelles, contrôle de la natalité et de la mortalité, etc.) qui sont en général le fait des politiques ou des économistes, ainsi que d'experts issus d'horizons les plus divers, semblent finalement avoir trouvé un écho dans des orientations récentes de l'anthropologie népalaise ${ }^{9}$.

Alors que les organisations et agences de développement sont pressées d'introduire dans leurs programmes les connaissances sociologiques et ethnologiques sur le Népal, des chercheurs en sciences sociales ont, de leur côté, cherché à intégrer les agents du développement (structures bureaucratiques de l'État ${ }^{10}$ ou ONG) et leurs actions dans leurs travaux. L'impératif de contextualisation, principe de l'ethnologie, a ceci d'avantageux qu'elle prévient le risque - parfois rencontré en sociologie - d'autonomiser ces formes d'organisation et d'action humanitaire : ce sont moins les ONG en elles-mêmes que ce que leur action contribue à dévoiler aux plans des ordres politiques, des dynamiques sociales et des configurations culturelles qui est intéressant pour l'ethnologie. C'est dans cette perspective que cet article traite de l'action d'une ONG (Tashi-Delek - Himalayan Health, ou TD-HH) et s'efforce de révéler et de questionner quelques logiques et enjeux de la mondialisation de l'idéologie et de l'action en faveur du développement, à partir d'une étude de cas localisée dans une région du nord-est du Népal.

\section{Contexte et enjeux de l'aide humanitaire au Népal}

$\mathrm{Au}$ Népal, la «mondialisation de l'aide humanitaire » s'est d'abord traduite par l'injection dans les politiques nationales des idéologies et des normes du dit développement. Comme s'il avait fallu que cette mondialisation - qui a été dans un second temps celle des actions concrètes et des transferts logistiques - soit précédée de conditions propices à sa réception. L'ouverture du pays, dans les années 1950 et après un siècle de fermeture aux influences extérieures, à une économie de marché globalisée et à la "modernité » occidentale, a été l'occasion d'une prise de conscience de son faible niveau de développement, à laquelle les gouvernements qui se sont succédés depuis lors se sont efforcés de remédier. L'action des institutions nationales népalaises a alors consisté en un découpage administratif du pays en cinq "régions de développement ", en la mise en place de plans quinquennaux affichant des objectifs de développement économique, social et sanitaire toujours plus ambitieux, consécutifs de l'adoption de standards de développement internationaux évalués par de grandes enquêtes à l'échelle nationale $^{11}$, mais également en la mise en 
place de programmes de développement sur le mode «top-down", dont l'application concrète incombe encore aux structures politiques locales (conseils ou panchayats de village), et qui ont été remaniés à diverses reprises (avec le glissement de programmes de développement simples "imposés" à des programmes "intégrés", plus axés sur la polyvalence des acteurs, la participation des communautés indigènes, le développement local et durable). Parallèlement, et avant que ces politiques de développement ne fassent la preuve de leur échec relatif, le Népal devait consentir à accepter l'aide financière, technique et matérielle d'autres nations (d'Inde, des États-Unis, de Russie et de Chine) tout autant que l'intervention des mouvements caritatifs ${ }^{12}$ ainsi que d'organisations internationales (en particulier l'OMS) qui se sont multipliées et diversifiées depuis les années 1950 (UNICEF, ONU, USAID, la Banque Mondiale, pour ne citer que les plus connues).

La position du Népal dans l'échelle mondiale de la pauvreté, quoiqu'elle varie d'année en année, continue de situer le royaume himalayen parmi les pays les plus pauvres du monde, récemment désigné comme le troisième pays le plus pauvre d'Asie, après l'Afghanistan et le Bhoutan. Cette affirmation, largement relayée par la presse et la littérature spécialisées, est régulièrement confirmée par la position du Népal dans les derniers rangs du classement effectué par le United Nations Development Program, qui prend, depuis 1990, comme outil de classement, le Human Development Index ${ }^{13}$. À l'exception des institutions gouvernementales et des grandes organisations internationales, les agents et acteurs du développement en activité au Népal ignorent souvent l'existence de ces rapports officiels: d'autres sources d'inspiration semblent motiver leur action. L'idéologie tiers-mondiste qui combine paradoxalement la fibre altruiste, le paternaliste d'un Occident dominateur, et son versant obscur, la culpabilité ${ }^{14}$, n'est pas l'unique raison de l'essor d'une aide au développement au Népal : s'y ajoute en effet une "attraction romantique $" 15$, nourrie aux images exotiques d'un Himalaya " secret " et " mystique ", et à la richesse naturelle et culturelle s'adjoint le constat d'une apparente misère, surtout rencontrée dans les campagnes. L'action humanitaire conduite au Népal semble influencée par le sentiment ambigu qui se dégage de l'union de ces représentations sous certains aspects antinomiques ${ }^{16}$.

\section{CSO et ONG au Népal}

L'une des particularités du Népal est le développement exponentiel de Civil Society Organizations (CSO) et d'Organisations Non Gouvernementales locales (ONG), parallèle à l'action d'ONG internationales (en nombre plus réduit). La première ONG fondée au Népal date de 1926. On ne comptait qu'une dizaine de CSO et d'ONG en 1960 , et elles n'étaient encore que 37 en 1977, à la date de fondation du Social Service National Coordination Council, qui allait devenir le Social Welfare Council, un organisme d'État chargé d'identifier et - jusqu'à un certain point seulement - de coordonner des actions qualifiées de sociales, culturelles ou humanitaires non gouvernementales menées sur le territoire national. Depuis une décennie, toutefois, le nombre de CSO et d'ONG au Népal a connu une croissance aussi rapide qu'inattendue : alors qu'elles étaient encore moins de 200 à la fin des années 1980, on en comptait plus de 8000 à la fin des années $1990^{17}$. Une première explication à ce développement fulgurant est d'ordre politique : l'avènement d'une monarchie parlementaire en 1990 a signifié la fin des restrictions qui s'appliquaient jusque-là aux associations. Dans un autre ordre d'idées, le développement récent et massif des CSO et des ONG au Népal peut être vu non pas comme un phénomène inédit, s'inscrivant au contraire dans la continuité d'une forme "traditionnelle» de solidarité, connue sous le nom de guthi (en népali), dont les CSO et les ONG ne sont que l'expression contemporaine, traduite dans la terminologie du «développement » et de l'« action sociale » qui s'est diffusée à l'échelle nationale via les acteurs de la coopération. Leur développement semble également correspondre à l'essor d'une "société civile» au Népal, dont les promoteurs et les acteurs soutiennent qu'elle remédie à l'incapacité du gouvernement népalais à répondre à des exigences exprimées par la population en matière d'égalité sociale, d'emploi, d'éducation et de prise en charge sanitaire $^{18}$ - lesquelles affectent principalement des catégories sociales plus exposées que les autres, notamment les basses couches sociales et les femmes, en particulier dans les milieux ruraux - voire de protection de l'environnement. Autrement formulé, l'émergence d'un secteur associatif, relais des actions humanitaires, participe à la fois d'une actualisation de formes antérieures et d'une innovation sur le plan de la solidarité.

Comme dans bien d'autres pays, les ONG (dans lesquelles on inclura désormais les CSO) représentent au Népal une constellation très diversifiée, qui recouvre un éventail très large d'actions et de projets. Elles peuvent être classées selon des critères de taille (individuelles ou organisations plus ou moins larges), d'origine (nationales ou internationales), de degré de légitimité (des initiatives privées jusqu'à celles qui bénéficient d'une reconnaissance officielle), de niveau d'organisation (aides ponctuelles et sporadiques jusqu'à de véritables programmes qui s'étendent dans le temps), d'échelle (action locale ou d'extension nationale). Bien plus que les critères morphologiques ou fonctionnels, c'est la nature de leur action qui distingue les ONG : les ONG locales s'orientent plutôt vers les questions de justice sociale et d'équité économique, de défense des droits sociaux, de préservation culturelle et plus récemment écologique (ce en quoi elles s'apparentent 
plutôt à des CSO), alors que les ONG internationales - qui ne sont pas toutes " occidentales " - demeurent plus fermement établies dans les domaines du développement économique, du transfert de technologie et de la santé. Toutes les ONG internationales ne sont néanmoins pas de grandes organisations, et l'action humanitaire se déploie également dans le cadre de petites ONG, dont TD-HH qui officie au Solukhumbu, l'un des soixantequinze districts du Népal, situé dans le nordest du pays, à la frontière tibétaine.

\section{Une ONG dans l'Himalaya népalais : Tashi-Delek - Himalayan Health}

\section{Localisation et rayon d'action}

Situé au nord-est de la vallée de Katmandou, le Solukhumbu est éloigné des grands centres urbains, majoritairement situés dans le centre et le sud du pays. Composé d'un ensemble de vallées et de montagnes de moyenne et de haute altitude, il est de plus difficile d'accès par les voies terrestres, et n'est desservi par les voies aériennes que grâce à la présence de deux petits aéroports. Cette situation, qui place ce district en marge de la géographie du développement national (concentré dans les zones urbaines ou à forte densité démographique), ne lui est pourtant pas totalement préjudiciable. En effet, le Solukhumbu est l'une des premières régions du Népal à s'être ouverte au tourisme, après la chute de la dynastie rana qui avait interdit l'accès au royaume népalais aux visiteurs étrangers (asiatiques compris) durant près d'un siècle. Depuis les années 1950, de nombreuses expéditions d'alpinistes se sont succédées pour gravir les pics montagneux situés dans cette région, et notamment l'Everest, le plus haut sommet du monde. L'exploit allait être réalisé en 1953 par le Néozélandais Sir Edmund Hillary, accompagné d'un sherpa dénommé Tenzin Norgay ${ }^{19}$ : ce qui allait pour longtemps ancrer les sherpas dans un imaginaire culturel et sportif occidental, les figurant comme de fervents bouddhistes, de chaleureux représentants d'une culture "authentique" et comme de valeureux alpinistes (ou "tigres des neiges ", ce qu'ils n'étaient pas véritablement avant cette époque $)^{20}$.

Par delà ses prouesses sportives, Hillary est considéré par les sherpas à la fois comme un bienfaiteur et comme un ardent défenseur de leur culture. Le célèbre alpiniste devait en effet fonder dès les années 1960 l'Himalayan Trust, une organisation dont les objectifs s'inscrivent dans des enjeux de développement économique, social et sanitaire, et dans une démarche de promotion et de préservation culturelle. Au plan sanitaire, l'Himalayan Trust est en effet à l'origine de la fondation du premier hôpital dans le Solu (dans le village de Phaphlu) dans la partie méridionale $\mathrm{du}$ district, et d'autres dispensaires de médecine occidentale dans divers villages du Khumbu, qui recouvre les régions septentrionales. Son action sera suivie quelques années plus tard par une autre organisation internationale, CARE, qui allait également contribuer au développement local des services de santé, puis par Himalayan Health qui allait s'associer à une ONG française, Tashi-Delek. Si l'action de l'organisation CARE s'est limitée au domaine de la santé, Himalayan Trust et Himalayan Health ont en revanche étendu leur action à la sphère éducative (création d'école, de programmes d'éducation sanitaire) et culturelle (via la promotion de formes d'expression culturelles locales - arts, danses, rites, artisanat, etc.).

L'association Tashi-Delek / Himalayan Health (TD-HH) a vu le jour en 1994. À l'origine se trouvent deux associations, fondées par deux docteurs en médecine, l'un français, Alain Cantero et l'autre sherpa, Mingmar Sherpa, le premier étant le fondateur de Tashi-Delek ${ }^{21}$, le versant français de l'association, le second étant quant à lui un ancien membre actif de l'Himalayan Trust et fondateur de l'Himalayan Health, sur le versant népalais. TD-HH est donc bicéphale et compte parmi ses principaux objectifs le développement d'un système biomédical dans les parties basse et moyenne du Solukhumbu - respectivement le Solu et le Pharak. Au registre de ses actions se trouvent l'identification des pathologies locales et l'étude des coutumes culturelles (notamment au plan sanitaire) préalables à l'implantation de la médecine occidentale, la construction d'hôpitaux mobilisant la population locale (dans plusieurs villages relativement éloignés les uns des autres : Karikhola, Kinja, et Nele), la formation et le recrutement de personnel dans cette même population, et des objectifs d'autogestion à moyen et à long termes par les institutions villageoises des dispositifs mis en place, qu'ils relèvent de l'action sanitaire ou de la promotion culturelle ${ }^{22}$. Hormis l'action en direction des populations sherpas et d'autres groupes ethniques qui sont installés dans le Solukhumbu, TD-HH réserve une partie de ses efforts à l'aide aux Tibétains - qui se sont massivement implantés dans le Solukhumbu à la suite de l'annexion du Tibet par le gouvernement communiste de Beijing - en s'associant au Men-Tsee-Khang, l'organisation qui fédère et diffuse la médecine tibétaine en Inde, au Népal et ailleurs. TD-HH apporte son soutien financier et logistique au dispensaire du camp de réfugiés tibétains de Chialsa (Solu) ainsi qu'au dispensaire mixte (de médecine occidentale et tibétaine) du monastère tibétain Thoutpen Choeling (Pharak). Dans sa démarche globale comme dans la chronologie de ses objectifs, TD-HH semblait avoir évité les écueils que rencontre généralement l'imposition d'un modèle «topdown ": écart trop grand entre les besoins locaux et les services proposés ${ }^{23}$, passivité de la population, "possession" (ownership) exclusive du programme de développement ${ }^{24}$. Néanmoins, et malgré d'évidents succès en matière sanitaire, TD-HH a été confrontée à des problèmes liés non pas à sa structure, ni, non plus, à sa dynamique, mais à son 
environnement. Je n'en mentionnerai ici que trois : le développement anarchique d'un sidehelp, la concurrence d'autres ONG, et l'inertie de la population locale.

\section{Tourisme et initiatives spontanées}

Si les organisations comme Himalayan Trust et TD-HH bénéficient d'une assez large visibilité au Solukhumbu, il existe une forme d'action parallèle nettement moins saisissable, mais tout aussi importante par son caractère massif et par le rôle singulier qu'elle joue dans le développement sanitaire local : l'initiative spontanée. Cette forme d'action humanitaire est une conséquence directe du tourisme. Une large partie de l'économie népalaise s'est en effet organisée autour de l'industrie d'un tourisme culturel et surtout sportif, comme en témoigne l'accroissement rapide des agences de trekking créées ou gérées par des sherpas à Katmandou ou la croissance d'un marché de l'artisanat (en particulier des artefacts religieux). Le tourisme, il faut le rappeler, est évalué au milieu des années 1990 à plus d'un demi million de visiteurs étrangers (dans un pays qui comptait alors environ 20 millions d'habitants), et, avant que la guérilla maoïste ne se déclare et ne fasse les dégâts que l'on connaît (plus de 6000 morts selon les sources officielles), les projections du gouvernement s'élevaient à un million de visiteurs potentiels dans les années 2000. Il engrangerait un cinquième du PNB.

L'un des effets empiriquement repérables du tourisme pourtant peu évoqué dans la littérature savante et qui se retrouve cette fois directement au plan de l'action humanitaire c'est d'avoir contribué, par l'augmentation des contacts entre Occidentaux et populations locales, à susciter des initiatives humanitaires spontanées et très localisées qui prennent le nom de side-help. C'est du moins sous ce vocable que les villageois désignent des aides ponctuelles qui prennent la forme de legs de médicaments ou de devises, plus rarement d'appareils médicaux, qui sont directement distribués à la population locale ou parfois aux dispensaires par des voyageurs au terme de leur trek (qui se séparent ainsi des médicaments transportés à leur propre usage et destination). Nul contrôle n'est exercé sur le side-help, dont le contenu (à l'exception des dons monétaires) est souvent d'une utilité très réduite (il répond initialement plus aux besoins des trekkeurs qu'à ceux des villageois). Les initiatives qui président au side-help ne se transforment pas toujours en actions concrètes ou en opérations organisées $^{25}$ : elles ne se substituent pas non plus aux aides d'organisations reconnues ou aux aides de l'État, mais viennent s'y adjoindre, d'où l'expression side-help qui signale l'ordre d'importance dans lequel sont conçues les actions durables (centrales) et les initiatives spontanées (périphériques) pour leurs principaux destinataires.

\section{Autonomie et dépendance relatives des ONG}

L'un des traits caractéristiques les plus couramment attribués aux ONG est leur indépendance par rapport aux structures de l'État ${ }^{26}$. Dans le contexte népalais, les rapports entre les ONG et l'État sont particulièrement ambivalents. Toutes les ONG devraient normalement être enregistrées auprès du Social Welfare Council, à plus forte raison si leur action touche au domaine de l'aide sanitaire, qui fait également l'objet d'un contrôle par le Ministère de la santé népalais. Cet impératif d'officialisation est néanmoins loin d'être toujours observé : une partie de l'action humanitaire - ONG ou actions spontanées - échappe ainsi au contrôle des institutions locales (les panchayats), et donc aux institutions nationales (les ministères concernés par la nature de l'action humanitaire). Et c'est par hasard que les fondateurs de TD-HH ont été informés de la fondation de dispensaires en divers points du Solukhumbu (en particulier dans les villages de Beni et de Ringmo), non loin des villages où sont déjà implantés des dispensaires. Après enquête, il s'avère que ces créations «indépendantes " résultent directement de la transformation d'initiatives spontanées en action plus durable : deux ONG distinctes (françaises) ont été créées spécialement à cette occasion. D'autres actions ont été récemment signalées (fin 2002) dans une autre partie du Solukhumbu : il s'agissait d'une campagne de délivrance de soins en direction des villageois accompagnée d'une distribution de médicaments par une ONG allemande, dont les membres étaient apparemment convaincus de pallier l'absence de services de santé dans la région. Si l'absence de démarches officielles auprès du Ministère de la santé a été un premier motif d'agacement exprimé par Mingmar Sherpa ${ }^{27}$ (représentant officiel de cette institution au Solukhumbu), d'autres raisons ont contribué à entraver toute possibilité de créer de bonnes relations entre les ONG anciennement et nouvellement implantées dans cette région : en premier lieu, le manque de concertation entre les différents acteurs de l'action humanitaire, méconnaissant souvent la présence des autres.

Les ONG ne sont pas les seules institutions auxquelles échoit la responsabilité de fournir aux populations locales des services de soin. Les objectifs de planification de l'État ont entraîné la fondation de Health Posts, postes avancés où sont dispensés des soins de première nécessité (en conformité avec le projet gouvernemental d'extension d'un Primary Health Care à l'échelle nationale). Les lieux de soin du Solukhumbu se partagent ainsi entre des Health Posts d'État, et des dispensaires et hôpitaux nongouvernementaux. Aucun acteur ne semble avoir véritablement pris ombrage de cette mitoyenneté, ni, par ailleurs, de l'évidente supériorité financière et logistique des services de santé établis par des ONG internationales ${ }^{28}$. Avec le temps (et sous l'impulsion des acteurs locaux, en particulier 
des responsables de TD-HH) s'est constituée une sorte de réseau de santé (aux relations plus ou moins formalisées) qui dirige les flux de patients d'un lieu de soin à un autre en fonction de la gravité de leur maladie. Les Health Posts gouvernementaux ne peuvent s'occuper que des affections légères: les médecins qui y officient orientent les patients atteints de pathologies plus lourdes vers les cliniques et dispensaires de TD-HH. Si le patient nécessite des soins plus importants, il est alors expédié à l'hôpital de l'Himalayan Trust qui dispose du matériel adéquat pour des interventions chirurgicales. Les cas les plus sérieux (notamment de tuberculose avancée) sont finalement rapatriés par voie aérienne dans l'un des hôpitaux gouvernementaux situés dans la vallée de Katmandou, où ils peuvent être traités dans des services de soins intensifs. Cette coopération, fruit à la fois d'une rationalisation des rapports entre ONG locales et d'ajustements plus empiriques, se trouve ainsi affectée par les ONG non intégrées dans le réseau sanitaire qui occasionnent, par leur action, des "dérivations", déviant les flux de patients du processus de prise en charge médicale adapté à la gravité de leur pathologie.

\section{Action humanitaire internationale : ajustements et incohérences}

Il résulte de leur présence simultanée et de leurs dynamiques différentes un développement "désordonné » des ONG qui se traduit, en premier lieu, par une concentration géographique des services de soin contraire à une logique de répartition rationnelle, un " maillage » territorial adapté à la distribution géographique et aux variations démographiques locales tel que le voudraient les organisations déjà implantées, et en second lieu par un évident manque de coordination entre les dispositifs de santé d'implantation ancienne et récente, respectivement, que l'existence d'une side-help incontrôlée vient encore contrarier. Au regard des faits exposés précédemment, on comprend mieux, dès lors, certaines caractéristiques saillantes du champ de l'aide sanitaire dans cette région du Népal, en l'occurrence : les demandes continuelles de la population locale pour une aide humanitaire qui semble intarissable et facile à obtenir; des relations entre ONG qui participent plus d'une superposition que d'une intégration, d'une concurrence que d'une coopération; la dispersion des ONG sur le territoire qui n'embrasse pas les contours d'une géographie de la pauvreté et du développement, mais qui ressortit à ses propres mécanismes et à la contingence des situations locales.

Pour autant, le constat d'une dispersion des ONG et d'une incohérence de leurs actions ne vaut que pour les institutions elles-mêmes, rapporté, qui plus est, à une conception idéale et normative de l'action humanitaire, qui serait à la fois méthodiquement organisée et dont la cohérence serait garantie par une rationalité minimale (dans la formulation et la réalisation des objectifs). Les populations locales demeurent en revanche assez étrangères à ces questions : pour les bénéficiaires de l'assistance humanitaire, la fameuse expression de Paul Feyerabend «tout est bon » est particulièrement pertinente, et pour poursuivre dans l'emprunt au célèbre philosophe des sciences, il convient d'ajouter " à partir du moment où ça marche ». Non qu'il faille voir dans l'accumulation des aides une sorte d'appétence calculatrice ou encore une insatiable soif de fonds et de services dispensés à des foules miséreuses : l'abondance des offres d'aide n'apparaît pas non plus comme une offense à la fierté des populations népalaises de ces moyennes montagnes mais comme une opportunité dont les villageois auraient tort de se priver, d'autant qu'elle paraît se légitimer en des termes culturellement acceptables.

En effet, la plupart des villages visités au cours de mes recherches ${ }^{29}$, localisés entre Phaphlu et Karikhola, comprennent au minimum une institution ou organisation locale qui bénéficie d'un parrainage étranger. Des monastères, écoles ou dispensaires sont financés par des associations étrangères, dont l'origine est souvent européenne (britannique, française, suisse, allemande notamment). Par ce biais, la présence occidentale semble s'être enchâssée dans certains domaines particulièrement importants de la vie sociale, culturelle et même religieuse tant et si bien qu'on en arrive à se demander, en extrapolant les observations locales à l'ensemble du pays, si le Népal ne représente pas tant une nation sous assistance économique et sanitaire (ce qui est une réalité), qu'une civilisation qui doit en partie sa pérennité au mécénat occidental. À partir d'un tel constat, il est facile de céder à la facilité en dénonçant une situation de subordination ou de domination dans une perspective néocoloniale : un examen plus approfondi des conditions locales suppose de réviser un tel jugement.

Les actions de sponsoring qui se développent en effet entre les ONG et les populations locales se comprennent en effet à la lumière des formes traditionnelles de solidarité et d'échange qui leur préexistent. Les ethnologues travaillant en pays sherpa ont révélé l'existence d'un système d'échange symbolique, le jindak, une forme de "fraternité rituelle» qui se superpose aux liens de parenté et qui engage les individus dans des rapports d'obligation et de réciprocité. Le jindak contribue à créer des relations (autres que contractuelles et résidentielles) entre lignées, familles et villages, tout autant qu'il associe les laïcs et les religieux dans une relation de "mécénat ${ }^{30}$. Pour l'anthropologue Vincanne Adams, le sponsoring occidental - en particulier celui d'Edmund Hillary - est d'autant mieux perçu, accepté voire recherché par les sherpas qu'il est interprété comme étant une extension du jindak à des personnes étrangères à la communauté ${ }^{31}$. En ce sens, le 
jindak (formulé localement sous le vocable de min) et les représentations culturelles qui lui sont associées (hospitalité, réciprocité, entraide) contribuent pleinement à favoriser l'activité de sponsoring à laquelle se livrent les Occidentaux au Solukhumbu. Il existe donc un cadre culturel à l'acceptation ou au rejet potentiel des «aides» (de quelque nature qu'elles soient) : et celui-ci, présentant déjà des propriétés fondamentales identiques à l'offre provenant de l'extérieur, favorise moins une transformation de la configuration locale, qu'une "greffe " de l'action humanitaire sur des principes sociaux et des représentations culturelles préexistantes. Ce constat ne doit pas être considéré comme la base utopique d'une "bonne» aide humanitaire, respectueuse des traditions locales et en conséquence "efficace». Elle n'est en effet pas contradictoire avec une toute autre réalité : les difficultés auxquelles les Occidentaux sont confrontés, lorsqu'ils entreprennent de mener une action sanitaire dans cette partie du Népal.

\section{Obstacles, difficultés et réajustements}

D'emblée, TD-HH, tout comme l'Himalayan Trust, allait reposer sur un partenariat entre les fondateurs et leurs collaborateurs locaux, auxquels ont rapidement incombé des responsabilités dans la gestion, l'organisation et le développement des services. C'est au double plan de la réalisation et de la durabilité de l'action sanitaire que s'est posée la question de l'engagement des habitants du Solu et du Pharak. Les fondateurs de TD-HH se sont adjoints les services des membres les plus actifs des communautés locales : d'une part, les détenteurs du pouvoir politique, et d'autre part, les jeunes villageois, plus éduqués, et plus enclins à induire ou à accompagner le changement dans leurs propres communautés.

Malgré l'existence de ces supports sociaux, l'accès à une autonomie logistique et financière des dispositifs de santé, qui était un des objectifs fondamentaux de TD-HH, s'est néanmoins heurtée à d'épineux problèmes. Le premier d'entre eux est le manque de moyens humains : il y a peu de personnel suffisamment qualifié pour dispenser les soins médicaux (et les formations offertes par les services locaux ne permettent pas de résoudre ce problème), les médecins népalais préférant exercer dans la vallée de Katmandou, qui leur offre à la fois de meilleurs revenus et des conditions de vie moins rudes que celles qu'ils connaissent dans la montagne. À ce manque de constance du personnel médical s'ajoute l'irrégularité des flux financiers qui ont été (et demeurent encore) largement tributaires des mécènes et donateurs français. Si la population reste en situation de dépendance par rapport à des sources françaises qui s'épuisent d'année en année, ses besoins ne cessent de s'accroître : les membres de TD$\mathrm{HH}$ ont alors dû consentir à un certain nombre d'ajustements et d'inflexions dans certains de leurs principes de base, notamment dans la gratuité des services et des médicaments. De même, sur une durée de presque dix ans, les actions "culturelles" entreprises par TD-HH ont été réduites au profit d'un recentrement sur des actions principalement sanitaires.

\section{$L$ ' inertie » de la population locale}

Le soupçon pèse parfois sur les ONG, notamment celles qui, paradoxalement, prônent le développement local, d'entretenir, par les conditions mêmes de leur action, cette situation de dépendance, en reproduisant, en quelque sorte, un état de contrôle de nature presque «impérialiste». Les observations empiriques révèlent toutefois que la situation n'est pas aussi simple : dans le cas de TD-HH, les statistiques de fréquentation des lieux de soin, et les données qualitatives (entretiens, observations ethnographiques) montrent que les habitants des vallées du Solu et du Pharak ont pleinement intégré l'usage des services de santé occidentaux dans leurs pratiques sanitaires. En revanche, les acteurs locaux, élus ou simples habitants, demeurent en situation de demandeurs, exprimant leurs motifs d'insatisfaction par rapport aux soins chaque fois que l'occasion leur est donnée, mais délaissant parallèlement les possibilités qui leur ont été données d'intervenir directement sur l'amélioration des soins. Mingmar Sherpa avait par exemple nourri le projet d'une participation au financement des dispositifs de santé de la part de chacun des utilisateurs, en affectant aux dispensaires le montant d'une journée de leur activité économique (qu'elle soit par ailleurs commerciale, agricole, artisanale ou dans l'industrie du tourisme) par mois : cette proposition n'a jamais été acceptée (ni par ailleurs totalement rejetée) par les villageois du Solukhumbu. L'ambition de développement local incluant la participation de la population aux projets proposés par TD-HH se heurte donc à une résistance passive mais implacable.

Les ONG au Solukhumbu accompagnent et même contribuent - de manière plutôt involontaire - à la mise en œuvre des actions sanitaires telles que formulées dans les programmes de développement nationaux. Si le gouvernement népalais a rencontré de nombreux problèmes s'agissant de l'extension de tels programmes à l'échelle du pays, les ONG ne sont finalement pas des acteurs beaucoup plus efficaces : elles rencontrent des problèmes identiques, auxquels viennent s'ajouter d'autres difficultés liées à leur statut et à leur position de relative autonomie, qui en font des entreprises concurrentielles dans le domaine de la santé, dont l'action est marquée d'un côté, par des performances souvent couronnées de succès, mais d'un autre côté, par la dispersion, la rivalité et l'incohérence. Le changement attendu dans les zones rurales - au moins au plan sanitaire - demeure lent et fastidieux. On attribue généralement l'échec des projets de développement à 
l'absence de participation de la part de la population locale ${ }^{32}$, aux faiblesses structurelles ou aux incohérences inhérentes aux institutions bureaucratiques qui sont censées les promouvoir et les impulser, aux difficultés de communication et de coordination entre les acteurs en présence (d'État ou non-gouvernementaux), ou encore à l'écart qui existe entre les projets de planification et les besoins réels des populations. D'autres facteurs sont à l'œuvre et sont à rechercher du côté de l'environnement de l'aide humanitaire, qui est complexe, fluctuant et désordonné. Si, de plus, on déplace la focale pour saisir non plus la dynamique des institutions ou la configuration des structures sociales, mais les systèmes de valeurs et les idéologies à l'œuvre du côté des populations elles-mêmes, alors des phénomènes tels que l'inertie de la population $\mathrm{du}$ Solukhumbu face aux actions de TD-HH peuvent s'expliquer en termes de "culture». Pour reprendre les mots de Dor Bahadur Bista, il existe une différence de "culture " entre les agences de développement et les ONG, d'un côté, et la population népalaise, de l'autre $^{33}$ : la première se caractérise par un activisme et un optimisme sans faille, la seconde, par un pessimisme et une léthargie inscrits plus généralement dans l'idée du fatalisme, dont les racines plongent au plus profond des valeurs culturelles (et surtout religieuses) du Népal. L'analyse de D. B. Bista vaut surtout pour l'hindouisme, responsable selon l'auteur de la sclérose sociale et culturelle du pays, et sa transposition à la situation d'autres régions du Népal, dont le Solukhumbu, à majorité bouddhiste, est des plus incertaines. Une autre raison, moins problématique, réside, selon Bista, dans l'attitude de "dépendance paternelle » (paternal dependency) que manifestent les Népalais - pénétrés de l'idée de pauvreté qui leur a été assignée et assénée - à l'endroit de l'aide internationale, et qui explique qu'ils la considèrent comme acquise de droit et ne dissimulent rien de leurs ressentiments si celle-ci n'est pas conforme à leur attentes ${ }^{34}$ tout en se gardant de jouer un rôle actif dans le développement.

Somme toute, les espoirs placés dans l'essor d'une société civile au Népal qui serait activement engagée dans le développement au titre non plus de "cible " de l'aide internationale, mais de véritable partenaire pour celle-ci, suppose, pour leurs plus ardents défenseurs, une réforme profonde de la nation népalaise, tant au plan des structures sociales qu'à celui des mentalités. À l'exception des milieux urbains les plus éduqués, les seuls à nourrir ce même projet sont les activistes maoïstes. Étrangement, les intellectuels progressistes népalais et les révolutionnaires armés (dont les relations ne sont pas vraiment de nature empathique) se retrouvent au moins sur un point: doter le peuple de la souveraineté. Mais au final, les deux parties se distinguent sur le but à atteindre : une démocratie pour les uns, une "démocratie populaire» pour les autres. Ce détour par le mouvement maoïste est l'occasion de mentionner l'impact de la "guerre de libération " qu'il a engagée depuis février 1996, sur l'action humanitaire. Le Solukhumbu n'a été que récemment affecté directement par la guérilla maoïste, qui ne s'y est répandue que vers les années 2000 et 2001. Favorables à la médecine occidentale au nom d'un rejet des "superstitions" que représentent les médecines locales (ayurvédiques et chamaniques), favorables, également, à l'aide internationale (mais sous certaines conditions), les maoïstes adoptent cette attitude paradoxale d'exiger, par la force, que les ONG versent un "impôt révolutionnaire ", ce qui a suscité une diminution considérable de l'activité humanitaire. Les combats entre les forces gouvernementales et maoïstes qui se sont déroulés dans le Solukhumbu ont, d'un autre côté, entraîné un ralentissement, voire un arrêt complet du fonctionnement des services de santé : l'hôpital du village de Ringmo en construction lors de mon séjour en 2001 était à l'abandon en 2002; la clinique de TD-HH construite dans la localité de Nele a été réquisitionnée par les maoïstes et leur sert occasionnellement de base de repli en situation de conflit armé ; enfin, en tant que représentants de l'État, les personnels travaillant dans les Health-Posts ont été menacés, parfois physiquement malmenés.

\section{Conclusion}

À la circonspection ou l'enthousiasme que suscite l'emploi du terme mondialisation en anthropologie, la préférence est ici allée à un examen rigoureux de cette notion à la lumière de données empiriques dont le recueil et le traitement restent le fruit d'une enquête ethnographique localisée. Dans ce sens, la mondialisation (du moins sous une forme particulière) a été soumise à un examen anthropologique que l'on pourrait qualifier de «classique ». En effet, alors que nombre de nos collègues invitent à repenser perpétuellement la discipline au gré des "nouveautés" (d'objets, de méthode, de perspective...), le terrain - comme fondement de la construction du savoir, malgré les variations de nature, de forme et de sens dont il se voit paré - nous semble demeurer le socle inamovible préalable à toute réflexion anthropologique. Avant de se lancer à corps perdu dans la vogue des différentes modes intellectuelles, faisons d'abord ce que nous savons faire le mieux, et constituons avant toute discussion cette armature indispensable à la réflexion que sont les faits.

Il apparaît sur cette base qu'une analyse des changements formulée en termes de mondialisation implique de conserver une perspective critique et relativiste, qui soulève ici trois questions. La première est celle de la genèse des changements observés par l'ethnologue. Faire de la mondialisation la source de toutes les transformations revient à mettre l'analyse anthropologique sous tutelle 
d'une de ces théories systémiques qui font florès actuellement (dite du world-system) qui lui confère une existence et un rôle déterminant sur un plan universel, et suppose de confirmer le slogan "rien n'échappe à la mondialisation ". Ces théories ultradéterministes, formulées par exemple sous la plume de Jonathan Friedman (1999) ou celle de Peter Beyer (1994), en offrant à l'analyse une perspective macroscopique, ne rendent pas totalement compte de ce qui se joue à l'échelle locale : l'inscription d'un processus de mondialisation particulier (ici, celui de l'aide humanitaire) dans une configuration locale complexe qui le transforme à son point d'arrivée et lors de sa "réception ". La seconde question est évidemment celle des impacts des processus de mondialisation. La nature et la profondeur des transformations que l'on observe ne confirment pas toujours l'idée qu'il faille voir là exclusivement l'œuvre de la mondialisation, cet expédient commode (à l'image de son précurseur la modernité) un peu rapidement et souvent trop facilement doté de la faculté d'impulser les mutations socioculturelles. Le tourisme, le développement économique et l'aide humanitaire sont, certes, clairement identifiés dans l'histoire du Népal comme des influences extérieures, inscrites dans des processus débordant très largement les frontières nationales, et sources de transformation pour une société en transition.

Ce sont néanmoins les acteurs locaux, inscrits dans des configurations sociales et politiques locales, porteurs de valeurs locales qui se confrontent à ces apports "mondiaux ", les évaluent au prisme de leurs propres attentes, de leurs propres projections sur leurs images de l'«étranger développé » (que recouvre le terme népali bikesh), en imposant aux actions humanitaires des orientations singulières. Et qui situent l'efficacité et le rayon d'action de ces dernières (déjà passablement brouillé par la confusion idéologique et pratique qui règne dans ce secteur d'activité) dans un espace social et politique des plus troubles et complexes (comprenant un maoïsme local qui est loin d'être une manifestation d'une plus large "mondialisation"), ce qui ajoute au procès d'une mondialisation aux moyens et aux effets uniformément altérants (sur le mode positif ou négatif). Car au final, il existe bien des conditions culturelles et idéologiques qui restreignent d'un côté, favorisent de l'autre l'acceptation et l'incorporation de ces fameux "flux " mondiaux dont Arjun Appadurai s'est fait le théoricien ${ }^{35}$, et des modalités particulières par lesquelles ces flux s'intègrent ou sont intégrés dans des dynamiques locales. Les données que l'on vient de présenter illustrent, certes, un cas qui ne saurait être considéré comme représentatif de l'ensemble des situations où s'observe cette mondialisation humanitaire et sanitaire. Mais sa singularité n'en fait pas moins un cas ethnographique dont on voudrait ici tirer une leçon: et celle-ci est plus d'ordre méthodologique que théorique.

La sagesse voudrait ainsi qu'une part fondamentale de l'analyse des faits dits de mondialisation soit positivement ramenée au local. Ce dernier n'est pas entendu comme simple produit méthodologique n'existant que par référence à la catégorie d'opposition par laquelle il prend sens - le global - ni, encore, comme un simple plan de manifestation où s'observent des processus d'extension mondiale dont il est trop souvent fait mention actuellement. On veut voir ici dans le «local» la matière, oserait-on dire, la réalité première de la vie des acteurs du contexte actuel (mondialisé ou pas) et en particulier de l'expérience ethnologique. Dire la mondialisation c'est, nous semble-t-il, d'abord s'efforcer de décrire, à des échelles différentes (pour autant que l'on puisse les atteindre en anthropologie), les processus et les effets qui sont supposés la constituer : c'est la construire plus que la postuler, en déceler les éventuels effets sous des formes discrètes ou plus manifestes. Mais c'est aussi reconnaître les lieux et les secteurs où son emploi s'avère relever d'une projection abusive ou une concession à la dernière mode.

1 Mauro F. Guillén, "Is Globalization Civilizing, Destructive or Feeble ? A Critique of Five Key Debates in the Social Science Litterature ", Annual Review in Sociology, 27 (2001), p.p. 235-260.

2 Cf. Mondialisation, les mots et les choses, GEMDEV, Paris : Karthala, 1999.

3 Je renvoie ici à l'article que j'avais consacré à cette question, dans une précédente livraison de cette revue, intitulé «Anthropologie, religion et modernité. Quelques réflexions sur le modernisme et le primitivisme des sciences de l'Homme", Parcours Anthropologiques, Lyon (CREA), n 4, 2004, p.p. 11-21.

4 Constat effectué il y a plus d'une dizaine d'années par Dilli R. Dahal, dans sa contribution : «Anthropology of the Nepal Himalaya, A Critical Appraisal ", in : C. Ramble, M. Brauen (Eds), Proceedings of the International Seminar on the Anthropology of the Tibet and the Himalaya, Zürich : Ethnological Museum of the University of Zürich, 1993, p. 55.

5 Pour le Solukhumbu, terrain sur lequel ont été recueillies les données présentées dans cet article, voir Hans Guldberg Axelsen, « The Sherpas in the Solu District. A Preliminary Report on Ethnographical Field Research in the Solu District of North-Eastern Nepal ", Det Kongelige Dankse Videnskabernes Selskab HistoriskFilosofiske Meddelelser, 47/7 (1977) p. 59 ; Vincanne Adams, "Dreams of a Final Sherpa", American Anthropologist, 99 (1), mars 1997, p. 86.

6 Rohit Kumar Nepali, "Culturally Appropriate Development. An Anthropological Study of Villages in Eastern Nepal », in : C. Ramble, M. Brauen (Eds), Proceedings of the International Seminar on the Anthropology of the Tibet and the Himalaya, op. cit., p. 238.

7 M. Allen et R.J. Fischer signalent que nombre d'anthropologues qui mènent des recherches au Népal sont euxmêmes impliqués dans des organismes ou des projets de développement, occupant parfois un véritable rôle de consultant. Même si elle demeure tout à fait conforme à des idéaux académiques, l'anthropologie du Népal ou de 
l'Himalaya produit également des connaissances de nature à être utilisée à des fins de développement. Michael Allen, R.J. Fischer, "Introduction an Acknowledgements ", in : Michael Allen (ed), Anthropology of Nepal. Peoples, Problems and Processes, op. cit., p. xvii. Ailleurs, James Fischer, spécialiste mondialement reconnu de la culture sherpa, assénait à l'inverse une critique particulièrement sévère à l'endroit des experts du développement, qu'il accuse de formuler des jugements hâtifs et péremptoires, fondés sur des séjours trop brefs sur le terrain. James Fischer, Sherpas. Reflection on Change in Himalayan Nepal, Berkeley, Los Angeles : University of California Press, 1990, pp. xxiv-xxv.

8 Comme c'est le cas, par exemple, d'Ellen Kristvik, infirmière volontaire au Népal engagée dans un programme de lutte contre la tuberculose, qui a conduit une recherche sur les médecines locales. Ellen Kristvik, Drums and Syringes. Patients and Healers in Combat Against TB Bacilli and Hungry Ghosts in the Hills of Nepal, Katmandu : EMR, 1999.

9 Cf. Les contributions d'anthropologie du développement dans le volume collectif dirigé par Michael Allen Anthropology of Nepal. Peoples, Problems and Processes, Kathmandu : Mandala Books, 1994.

${ }^{10}$ Comme l'a fait Judith Justice au Népal. Judith Justice, Policies, Plans \& People. Foreign Aid and Health Development, Berkeley, Los Angeles, Londres : University of California Press, 1986.

${ }^{11}$ Parmi elles, il faut citer le Nepal Fertility Family Planning and Health Survey (1991) et le Nepal Living Standards Survey (1996) qui ont produit des données particulièrement importantes sur l'état sanitaire du pays.

12 Comme les jeunes Peace Corps Volunteers américains.

${ }^{13}$ La définition du HDI répond à la nécessité d'une évaluation comparative du « développement des sociétés humaines" et de la mesure des écarts de développement entre les nations. Il est calculé à partir de la corrélation de trois indicateurs : la "longévité ", la « connaissance ", et le «standard de vie " (PNB per capita ajusté au coût de la vie locale), lesquels sont construits à partir de critères empiriquement mesurables du développement (espérance de vie, années effectives de scolarité, revenu, etc.). Malgré les diverses critiques dont il a fait l'objet, le HDI sert encore au classement de 173 pays selon leur degré de développement. Cf. Shyam THAPA, "The Human Development Index : A Portrait of the 75 Districts in Nepal ", Asia-Pacific Population Journal, vol. 10, $\mathrm{n}^{\circ} 2$ (1995), p.p. 3-14.

${ }^{14}$ Cf. L'analyse incisive offerte par Pascal BRUCKNER, Le sanglot de l'homme blanc. Tiers-monde, culpabilité, haine de soi, Paris : Seuil, 1983.

15 J. Fischer, op. cit., p. 2.

16 Judith Justice, op. cit., p. 9.

17 Jagdish Ghimire, "NGO Developement in Nepal», in : B. B. Swar, S. R. Naupane, S. K. Basnet, (Eds.), Social Development and INGOs Activities in Nepal, 1999, Katmandou : Community Development Service Association, 1999 , p. 5.

${ }^{18}$ Cf. Dev Raj Dahal, Civil Society in Nepal. Opening the Ground for Questions, Kathmandu : Center for Development \& Governance, 2001.

19 Dont origine ethnique a d'ailleurs récemment fait l'objet d'une controverse. Il aurait en effet été un Tibétain et non un sherpa de "pure extraction".

${ }^{20}$ Vincanne Adams, Tigers of the Snow and Other Virtual Sherpas, Princeton : Princeton University Press, 1996.

${ }^{21}$ Expression supposée être une formule de salutation en langue tibétaine, mais qui a pour origine un usage rituel.

${ }^{22}$ Une action similaire dans sa réalisation et dans ses objectifs a également démarré en 2002, sous l'égide de Tashi Delek, en Mongolie.

${ }^{23}$ R. K. Nepali, «Culturally Appropriate Development...» op. cit., p. 238.

${ }^{24}$ Dev Raj Dahal, op.cit., p. 82.

${ }^{25}$ L'action de E. Hillary revêt sous cet angle la forme exemplaire d'un processus à l'œuvre dans l'action humanitaire au Népal : une démarche à l'origine individuelle, spontanée et fortuite qui s'est institutionnalisée.

${ }^{26}$ Centre Tricontinental, Editorial, Les ONG : instruments du néo-libéralisme ou alternatives populaires ? Paris : L'Harmattan, 1998, p. 5.

27 « Si je viens en France» affirmait-il en janvier 2003 « et que je veux exercer mon métier de médecin, je devrai faire des démarches auprès des institutions françaises, sinon mon activité serait illégale, n'est-ce pas ? et bien, il en va de même au Népal. Il n'y a donc aucune raison pour que les médecins étrangers exercent ici s'ils n'en ont pas reçu l'autorisation " (entretien personnel).

${ }^{28}$ La disproportion dans les montants financiers bruts attribués à la santé par les organismes sus-cités n'est pas seule responsable de ces différences de financements : cette différence tient également à la concentration des moyens attribués à des actions locales par les ONG, alors que les organismes d'État doivent répartir les leurs à l'échelle de la nation entière.

${ }^{29}$ Effectuées au cours de trois missions en 1999, 2001 et plus récemment en 2002-2003.

${ }^{30}$ Les sherpas étant bouddhistes, leurs religieux (les lamas) sont tenus de respecter un certain nombre de règles et de vœux. À la différence des lamas « de village », qui, nonobstant leur statut, mènent une vie presque ordinaire de paysan, les lamas de monastères sont supposés se livrer à des pratiques ascétiques et être affranchis de toute activité économique. Ce renoncement, qui n’est en vérité jamais totalement réalisé, installe les lamas et les laïcs dans une relation d'échange réciproque : les premiers offrant leurs compétences en matière de religion et de performance cultuelle, les seconds fournissant aux premiers les biens et aliments dont ils ont besoin.

31 Vincanne Adams, Dreams of a Final Sherpa, op. cit., p. 91.

${ }^{32}$ Qui tient plus du «slogan " que de la réalité selon R. K. Nepali, Culturally Appropriate Development... op. cit., p. 238.

33 Dor Bahadur Bista, Fatalism and Development. Nepal's Struggle for Modernization, sixième édition, Himayatnagar (Inde) : Orient Longman Limited, 1994, p. 130.

34 Id., p. 136.

35 Arjun Appadurai «Disjuncture and Difference in the Global Cultural Economy ", Theory, Culture and Society, vol. 7, 1990, réédité sous le même titre dans : Featherstone Mike (Ed.), Global Culture. Nationalism, Globalization and Modernity, $8^{\text {ème }}$ édition, London, Thousands Oaks, New Delhi : Sage Publications, 1999 (1990), p.p. 295-310. 